\title{
Analysis of Lossy Dielectric Objects with the Multilevel Fast Multipole Algorithm
}

\author{
Özgür Ergül \\ Department of Mathematics and Statistics \\ University of Strathclyde, Glasgow, UK \\ Email: ozgur.ergul@strath.ac.uk
}

\author{
Levent Gürel ${ }^{1,2}$ \\ ${ }^{1}$ Department of Electrical and Electronics Engineering \\ ${ }^{2}$ Computational Electromagnetics Research Center (BiLCEM) \\ Bilkent University, Bilkent, Ankara, Turkey \\ Email: lgurel@bilkent.edu.tr
}

\begin{abstract}
Rigorous solutions of electromagnetics problems involving lossy dielectric objects are considered. Problems are formulated with two recently developed formulations, namely, the combined-tangential formulation (CTF) and the electric and magnetic current combined-field integral equation (JMCFIE), and solved iteratively using the multilevel fast multipole algorithm (MLFMA). Accuracy and efficiency of solutions are compared for different objects and conductivity values. We show that iterative solutions of CTF are significantly accelerated as the conductivity increases and CTF becomes a good alternative to JMCFIE in terms of efficiency. Considering also its high accuracy, CTF seems to be a suitable formulation for the analysis of lossy dielectric objects.
\end{abstract}

\section{INTRODUCTION}

Homogeneous dielectric objects can be formulated rigorously with the surface integral equations that are based on the equivalence principle [1]-[9]. Numerical solutions of surface formulations require the discretization of surfaces between dielectric regions. Hence, they are suitable to formulate large dielectric objects, whose solutions can be impractical using volume formulations. It is also important to extend surface formulations to more realistic problems, such as those involving lossy objects with nonzero conductivity. Previously, lossy dielectric objects have been formulated with various surface integral equations, such as the Poggio-Miller-ChangHarrington-Wu-Tsai (PMCHWT) formulation [1]-[2],[6] and CFIE [3],[4]. Acceleration methods, such as the multilevel fast multipole algorithm (MLFMA) [3],[4], have been used for efficient solutions of large-scale problems involving lossy dielectric objects. However, a new set of integral-equation formulations has been proposed recently for improved solutions of dielectric objects [5],[7]-[9]. These novel formulations were also applied to lossy dielectric objects; but, they have not been investigated for large problems that are solved iteratively.

In this study, we consider the solution of relatively large electromagnetics problems involving lossy dielectric objects. Problems are formulated with the combined tangential formulation (CTF) [7] and the electric and magnetic current combined-field integral equation (JMCFIE) [5]. Solutions are performed iteratively using MLFMA. We show that, as the conductivity increases, CTF solutions are significantly accelerated such that CTF becomes a good alternative to JMCFIE in terms of efficiency. Since it is also more accurate than JMCFIE, CTF seems a suitable formulation for rigorous solu- tions of electromagnetics problems involving lossy dielectric objects.

\section{INTEGRAL-EQUATION FORMULATIONS}

Discretization of surface integral equations for homogeneous dielectric objects leads to $2 N \times 2 N$ dense matrix equations in the form of

$$
\left[\begin{array}{cc}
\overline{\boldsymbol{Z}}^{(11)} & \overline{\boldsymbol{Z}}^{(12)} \\
\overline{\boldsymbol{Z}}^{(21)} & \overline{\boldsymbol{Z}}^{(22)}
\end{array}\right] \cdot\left[\begin{array}{l}
\boldsymbol{x} \\
\boldsymbol{y}
\end{array}\right]=\left[\begin{array}{l}
\boldsymbol{v}^{(1)} \\
\boldsymbol{v}^{(2)}
\end{array}\right]
$$

where $\overline{\boldsymbol{Z}}^{(a b)}$ for $a, b=1,2$ are $N \times N$ partitions, whereas $\boldsymbol{x}$ and $\boldsymbol{y}$ are vectors of $N$ elements involving the coefficients for the equivalent electric and magnetic currents, respectively. For JMCFIE, matrix elements (interactions of basis and testing functions) are derived as

$$
\begin{aligned}
Z_{m n}^{(11)} & =\alpha \int_{S_{m}} d \boldsymbol{r} \boldsymbol{t}_{m}(\boldsymbol{r}) \cdot\left(\mathcal{T}_{o}+\mathcal{T}_{i}\right)\left\{\boldsymbol{b}_{n}\right\}(\boldsymbol{r}) \\
& +(1-\alpha) \int_{S_{m}} d \boldsymbol{r} \boldsymbol{t}_{m}(\boldsymbol{r}) \cdot \hat{\boldsymbol{n}} \times\left(\mathcal{K}_{o}-\mathcal{K}_{i}\right)\left\{\boldsymbol{b}_{n}\right\}(\boldsymbol{r}) \\
& -(1-\alpha) \int_{S_{m}} d \boldsymbol{r} \boldsymbol{t}_{m}(\boldsymbol{r}) \cdot \boldsymbol{b}_{n}(\boldsymbol{r}) \\
Z_{m n}^{(12)} & =(1-\alpha) \int_{S_{m}} d \boldsymbol{r} \boldsymbol{t}_{m}(\boldsymbol{r}) \cdot \hat{\boldsymbol{n}} \times\left(\eta_{o}^{-1} \mathcal{T}_{o}-\eta_{i}^{-1} \mathcal{T}_{i}\right)\left\{\boldsymbol{b}_{n}\right\}(\boldsymbol{r}) \\
& -\alpha \int_{S_{m}} d \boldsymbol{r} \boldsymbol{t}_{m}(\boldsymbol{r}) \cdot\left(\eta_{o}^{-1} \mathcal{K}_{o}+\eta_{i}^{-1} \mathcal{K}_{i}\right)\left\{\boldsymbol{b}_{n}\right\}(\boldsymbol{r}) \\
& -\frac{1}{2} \alpha\left(\eta_{o}^{-1}-\eta_{i}^{-1}\right) \int_{S_{m}} d \boldsymbol{r} \boldsymbol{t}_{m}(\boldsymbol{r}) \cdot \hat{\boldsymbol{n}} \times \boldsymbol{b}_{n}(\boldsymbol{r}) \\
Z_{m n}^{(21)} & =-(1-\alpha) \int_{S_{m}} d \boldsymbol{r} \boldsymbol{t}_{m}(\boldsymbol{r}) \cdot \hat{\boldsymbol{n}} \times\left(\eta_{o} \mathcal{T}_{o}-\eta_{i} \mathcal{T}_{i}\right)\left\{\boldsymbol{b}_{n}\right\}(\boldsymbol{r}) \\
& +\alpha \int_{S_{m}} d \boldsymbol{r} \boldsymbol{t}_{m}(\boldsymbol{r}) \cdot\left(\eta_{o} \mathcal{K}_{o}+\eta_{i} \mathcal{K}_{i}\right)\left\{\boldsymbol{b}_{n}\right\}(\boldsymbol{r}) \\
& +\frac{1}{2} \alpha\left(\eta_{o}-\eta_{i}\right) \int_{S_{m}} d \boldsymbol{r} \boldsymbol{t}_{m}(\boldsymbol{r}) \cdot \hat{\boldsymbol{n}} \times \boldsymbol{b}_{n}(\boldsymbol{r})
\end{aligned}
$$

where $\alpha=[0,1]$ is the combination parameter of JMCFIE, $\hat{\boldsymbol{n}}$ is the unit normal vector at the testing point, and $\eta_{u}=$ $\left(\mu_{u} / \epsilon_{u}\right)^{1 / 2}$ represents the intrinsic impedance of the outer $(u=o)$ and inner $(u=i)$ media. Integro-differential $\mathcal{K}$ and 
$\mathcal{T}$ operators are applied to the basis function as

$$
\begin{aligned}
\mathcal{T}_{u}\left\{\boldsymbol{b}_{n}\right\}(\boldsymbol{r}) & =i k_{u} \int_{S_{n}} d \boldsymbol{r}^{\prime} \boldsymbol{b}_{n}\left(\boldsymbol{r}^{\prime}\right) g_{u}\left(\boldsymbol{r}, \boldsymbol{r}^{\prime}\right) \\
& +\frac{i}{k_{u}} \int_{S_{n}} d \boldsymbol{r}^{\prime} \nabla^{\prime} \cdot \boldsymbol{b}_{n}\left(\boldsymbol{r}^{\prime}\right) \nabla g_{u}\left(\boldsymbol{r}, \boldsymbol{r}^{\prime}\right) \\
\mathcal{K}_{u}\left\{\boldsymbol{b}_{n}\right\}(\boldsymbol{r}) & =\int_{\mathrm{PV}, S_{n}} d \boldsymbol{r}^{\prime} \boldsymbol{b}_{n}\left(\boldsymbol{r}^{\prime}\right) \times \nabla^{\prime} g_{u}\left(\boldsymbol{r}, \boldsymbol{r}^{\prime}\right),
\end{aligned}
$$

where PV indicates the principal value of the integral, $k_{u}=$ $\omega\left(\epsilon_{u} \mu_{u}\right)^{1 / 2}$ is the wavenumber, and

$$
g_{u}\left(\boldsymbol{r}, \boldsymbol{r}^{\prime}\right)=\frac{\exp \left(i k_{u}\left|\boldsymbol{r}-\boldsymbol{r}^{\prime}\right|\right)}{\left|\boldsymbol{r}-\boldsymbol{r}^{\prime}\right|}
$$

denotes the homogeneous-space Green's function. In (2)-(6), $S_{m}$ and $S_{n}$ represent the spatial supports of $m$ th testing function $\boldsymbol{t}_{m}(\boldsymbol{r})$ and $n$th basis function $\boldsymbol{b}_{n}(\boldsymbol{r})$, respectively. Elements of the right-hand-side vector in (1) are derived as

$$
\begin{aligned}
v_{m}^{(1)} & =-(1-\alpha) \int_{S_{m}} d \boldsymbol{r} \boldsymbol{t}_{m}(\boldsymbol{r}) \cdot \hat{\boldsymbol{n}} \times \boldsymbol{H}^{i n c}(\boldsymbol{r}) \\
& -\alpha \eta_{o}^{-1} \int_{S_{m}} d \boldsymbol{r} \boldsymbol{t}_{m}(\boldsymbol{r}) \cdot \boldsymbol{E}^{i n c}(\boldsymbol{r}) \\
v_{m}^{(2)} & =(1-\alpha) \int_{S_{m}} d \boldsymbol{r} \boldsymbol{t}_{m}(\boldsymbol{r}) \cdot \hat{\boldsymbol{n}}(\boldsymbol{r}) \times \boldsymbol{E}^{i n c}(\boldsymbol{r}) \\
& -\alpha \eta_{o} \int_{S_{m}} d \boldsymbol{r} \boldsymbol{t}_{m}(\boldsymbol{r}) \cdot \boldsymbol{H}^{i n c}(\boldsymbol{r}),
\end{aligned}
$$

where $\boldsymbol{E}^{i n c}$ and $\boldsymbol{H}^{i n c}$ represent the electric and magnetic fields created by external sources in the outer medium extending to infinity. For JMCFIE, we use $\alpha=0.5$ in (2)-(4) and (8)-(9). For CTF, we use $\alpha=1.0$ and some of the integral are not required. For both formulations, $\overline{\boldsymbol{Z}}^{(11)}=\overline{\boldsymbol{Z}}^{(22)}$, i.e., the diagonal partitions are identical.

\section{Solutions with the Multilevel Fast Multipole ALGORITHM}

Electromagnetics problems formulated with JMCFIE and CTF can be solved iteratively with MLFMA [8]. Interactions between distant basis and testing functions are factorized, diagonalized, and calculated efficiently in three stages, namely, aggregation, translation, and disaggregation. Radiation pattern of $n$th basis function $\boldsymbol{b}_{n}(\boldsymbol{r})$ and receiving pattern of $m$ th testing function $\boldsymbol{t}_{m}(\boldsymbol{r})$ with respect to a reference point $\boldsymbol{r}_{C}$ can be written as

$$
\begin{aligned}
\boldsymbol{R}_{n}\left(\boldsymbol{r}_{C}, \boldsymbol{k}_{u}\right) & =\gamma_{n}(\overline{\boldsymbol{I}}-\hat{\boldsymbol{k}} \hat{\boldsymbol{k}}) \cdot \boldsymbol{S}_{n}^{-}\left(\boldsymbol{r}_{C}, \boldsymbol{k}_{u}\right) \\
\boldsymbol{R}_{m}^{(11, u)}\left(\boldsymbol{r}_{C}, \boldsymbol{k}_{u}\right) & =\boldsymbol{R}_{m}^{(22, u)}\left(\boldsymbol{r}_{C}, \boldsymbol{k}_{u}\right) \\
& =\alpha \gamma_{m}(\overline{\boldsymbol{I}}-\hat{\boldsymbol{k}} \hat{\boldsymbol{k}}) \cdot \boldsymbol{S}_{m}^{+}\left(\boldsymbol{r}_{C}, \boldsymbol{k}_{u}\right) \\
& -(1-\alpha) \hat{\boldsymbol{k}} \times \boldsymbol{S}_{m}^{\times n}\left(\boldsymbol{r}_{C}, \boldsymbol{k}_{u}\right) \\
\boldsymbol{R}_{m}^{(12, u)}\left(\boldsymbol{r}_{C}, \boldsymbol{k}_{u}\right) & =(1-\alpha) \eta_{u}^{-1}(\overline{\boldsymbol{I}}-\hat{\boldsymbol{k}} \hat{\boldsymbol{k}}) \cdot \boldsymbol{S}_{m}^{\times n}\left(\boldsymbol{r}_{C}, \boldsymbol{k}_{u}\right) \\
& +\alpha \gamma_{m} \eta_{u}^{-1} \hat{\boldsymbol{k}} \times \boldsymbol{S}_{m}^{+}\left(\boldsymbol{r}_{C}, \boldsymbol{k}_{u}\right) \\
\boldsymbol{R}_{m}^{(21, u)}\left(\boldsymbol{r}_{C}, \boldsymbol{k}_{u}\right) & =-(1-\alpha) \eta_{u}(\overline{\boldsymbol{I}}-\hat{\boldsymbol{k}} \hat{\boldsymbol{k}}) \cdot \boldsymbol{S}_{m}^{\times n}\left(\boldsymbol{r}_{C}, \boldsymbol{k}_{u}\right) \\
& -\alpha \gamma_{m} \eta_{u} \hat{\boldsymbol{k}} \times \boldsymbol{S}_{m}^{+}\left(\boldsymbol{r}_{C}, \boldsymbol{k}_{u}\right)
\end{aligned}
$$

where $\gamma_{m}= \pm 1$ and $\gamma_{n}= \pm 1$ are determined by the orientation of the testing and basis functions, and

$$
\begin{aligned}
\boldsymbol{S}_{n}^{-}\left(\boldsymbol{r}_{C}, \boldsymbol{k}_{u}\right) & =\int_{S_{n}} d \boldsymbol{r} \exp \left[-i \boldsymbol{k}_{u} \cdot\left(\boldsymbol{r}-\boldsymbol{r}_{C}\right)\right] \boldsymbol{b}_{n}(\boldsymbol{r}) \\
\boldsymbol{S}_{m}^{+}\left(\boldsymbol{r}_{C}, \boldsymbol{k}_{u}\right) & =\int_{S_{m}} d \boldsymbol{r} \exp \left[i \boldsymbol{k}_{u} \cdot\left(\boldsymbol{r}-\boldsymbol{r}_{C}\right)\right] \boldsymbol{t}_{m}(\boldsymbol{r}) \\
\boldsymbol{S}_{m}^{\times n}\left(\boldsymbol{r}_{C}, \boldsymbol{k}_{u}\right) & =\int_{S_{m}} d \boldsymbol{r} \exp \left[i \boldsymbol{k}_{u} \cdot\left(\boldsymbol{r}-\boldsymbol{r}_{C}\right)\right] \boldsymbol{t}_{m}(\boldsymbol{r}) \times \hat{\boldsymbol{n}}
\end{aligned}
$$

are the Fourier transforms of the functions. We note that the receiving pattern depends on the matrix partition, but the radiation pattern does not. Radiation and receiving patterns of basis and testing functions are connected through a set of shift and translation functions (depending on the positions of basis and testing functions) to perform the electromagnetic interactions between the functions.

\section{NUMERICAL EXAMPLES}

For comparisons, we consider the solution of scattering problems involving a lossy dielectric sphere of radius $0.3 \mathrm{~m}$ located in free space. The sphere is illuminated by a plane wave propagating in the $z$ direction with the electric field polarized in the $x$ direction at $1 \mathrm{GHz}$ and $3 \mathrm{GHz}$. For numerical solutions, surfaces are discretized with the RWG functions on $\lambda_{o} / 10$ triangles, where $\lambda_{o}=2 \pi / k_{o}$ is the wavelength in free space. The relative permittivity of the sphere is selected as 2.0 , while its conductivity changes from $0.001 \mathrm{~S} / \mathrm{m}$ to $10 \mathrm{~S} / \mathrm{m}$. Problems are formulated with JMCFIE and CTF discretized with 7446 and 65,724 unknowns. Both near-field and far-field interactions are computed with maximum $1 \%$ error.

Fig. 1 presents the number of conjugate-gradientsquared (CGS) iterations for $10^{-3}$ residual error with respect to the conductivity. We observe that iterative solutions improve as the conductivity increases, particularly for CTF. This is because matrix equations become diagonally dominant due to the contributions from the lossy medium. For JMCFIE, acceleration is observed from $\sigma=0$ to $0.1 \mathrm{~S} / \mathrm{m}$, followed by a deceleration to $\sigma=0.1 \mathrm{~S} / \mathrm{m}$ to $10.0 \mathrm{~S} / \mathrm{m}$. This is due to the unbalanced non-diagonal partitions of the matrix equations for large contrasts [9]. In general, as the conductivity increases, CTF solutions become comparable to JMCFIE solutions in terms of efficiency.

Fig. 1 also shows the relative error in the far-zone electric field with respect to the conductivity. Considering the error in matrix elements, the target error ceiling in numerical solutions is $1 \%$, which is also indicated in the plot. In order to find the relative error, the far-zone co-polar electric field, i.e.,

$$
e[n]=\lim _{r \rightarrow \infty}\left\{r E_{\theta}(r, \theta, \phi)\right\},
$$

is calculated and sampled at $0.5^{\circ}$ intervals on the $z$ - $x$ plane. Comparing the computational values with analytical Mieseries results, the error is defined as

$$
\epsilon=\frac{\left\|e_{\text {computational }}-e_{\text {analytical }}\right\|_{2}}{\left\|e_{\text {analytical }}\right\|_{2}},
$$



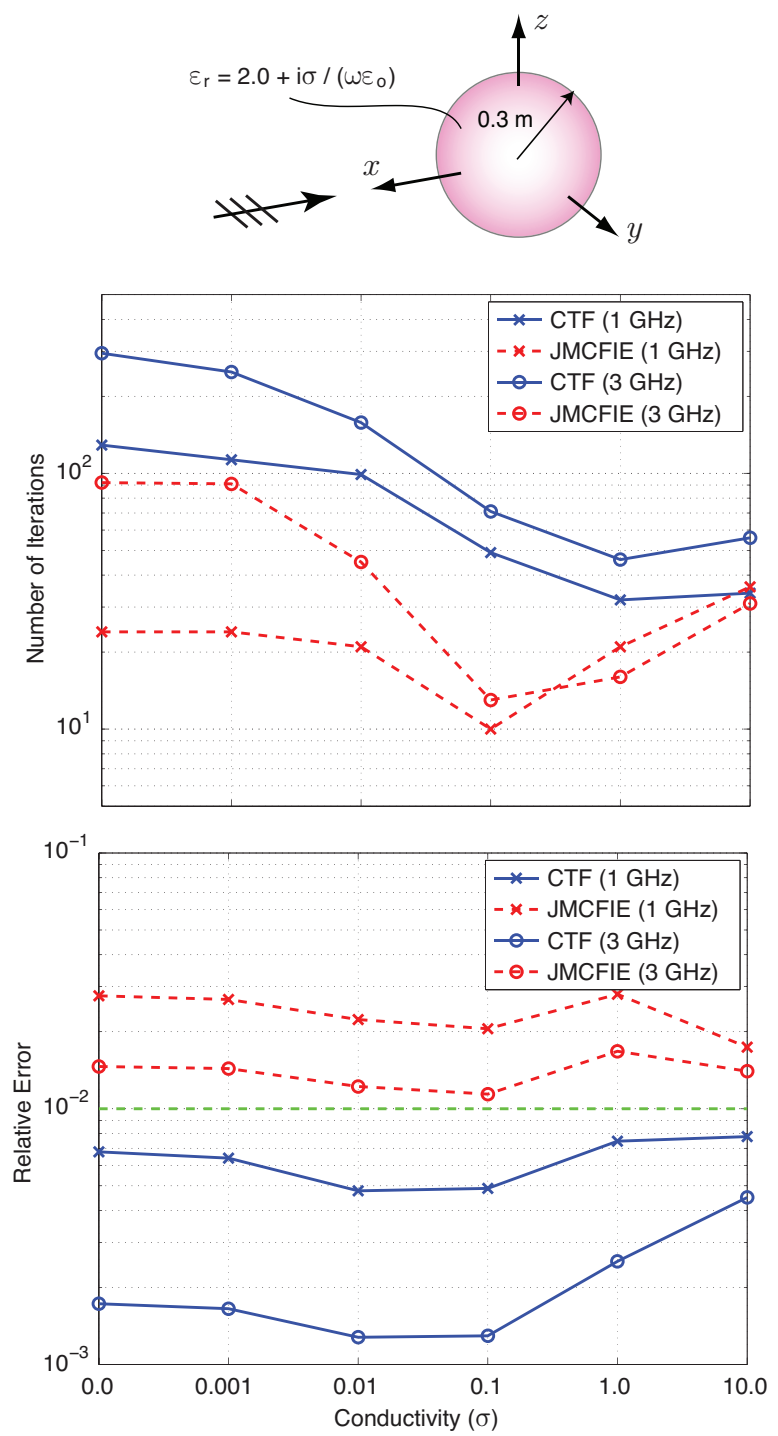

Fig. 1. Number of iterations and relative error in computational results for scattering problems involving a sphere of radius $0.3 \mathrm{~m}$ at $1 \mathrm{GHz}$ and $3 \mathrm{GHz}$. The relative permittivity of the sphere is 2.0 , while its conductivity changes from 0 to $10.0 \mathrm{~S} / \mathrm{m}$.

where $\|\cdot\|_{2}$ represents the 2-norm. As depicted in Fig. 1, the target relative error is not achieved if JMCFIE is used to formulate the problem, whereas CTF results are in the desired level of accuracy. Fig. 2 presents the bistatic radar cross section (RCS) of the sphere at $1 \mathrm{GHz}$ for different conductivity values from $0.001 \mathrm{~S} / \mathrm{m}$ to $1.0 \mathrm{~S} / \mathrm{m}$. We observe that CTF results perfectly agree with the analytical Mie-series solutions.

To further investigate the solution of problems formulated with CTF, we consider a periodic arrangement of five rectangular slabs located in free space, as depicted in Fig. 3. The structure is illuminated by a Hertzian dipole located at $z=3 \mathrm{~cm}$ at $20 \mathrm{GHz}$. Discretization with $\lambda_{o} / 10$ triangles leads to matrix equations involving 38,700 unknowns. The relative permittivity and the conductivity of the structure is 4.8 and
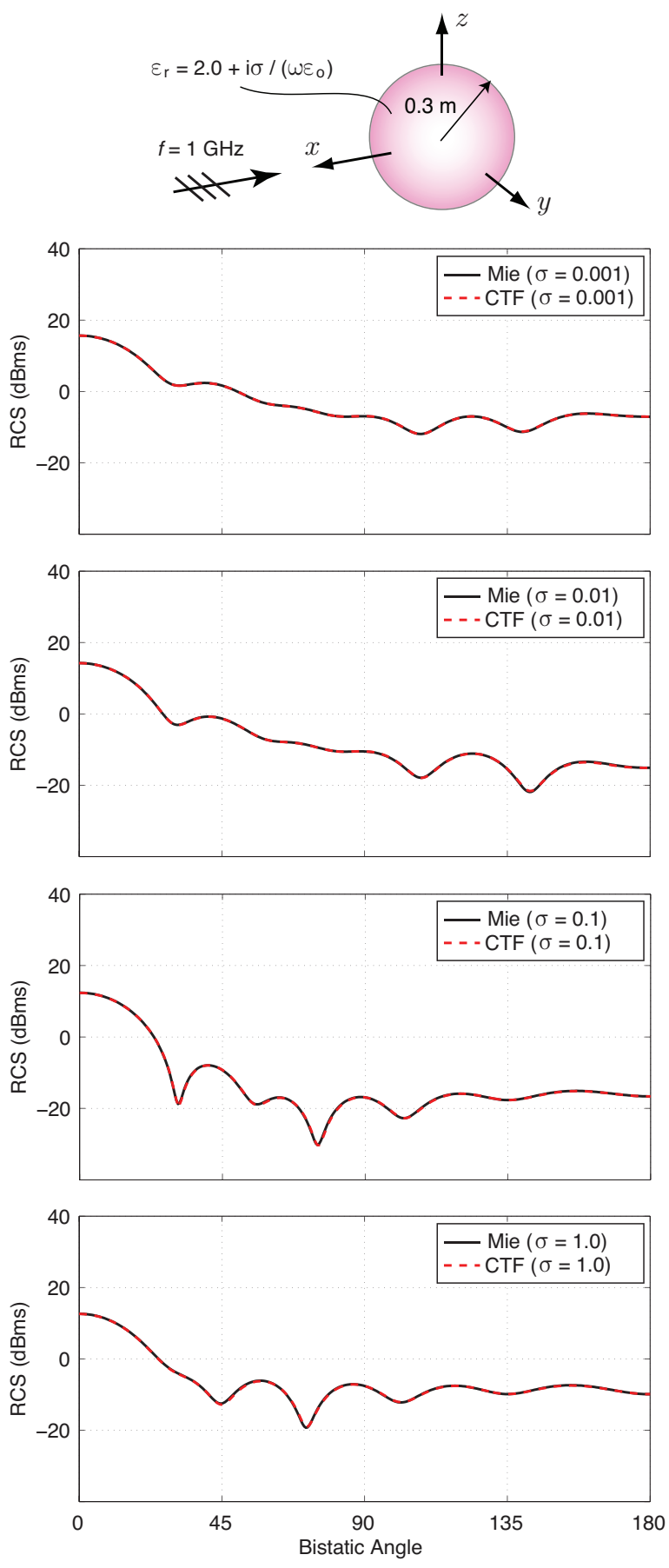

Fig. 2. Bistatic RCS of the sphere of radius $0.3 \mathrm{~m}$ at $1 \mathrm{GHz}$. Bistatic RCS is sampled on the $z-x$ plane from $0^{\circ}$ to $180^{\circ}$, where $0^{\circ}$ corresponds to the forward-scattering direction. The relative permittivity of the sphere is 2.0 , while its conductivity changes from 0.001 to $1.0 \mathrm{~S} / \mathrm{m}$.

$10 \mathrm{~S} / \mathrm{m}$, respectively. Fig. 3 illustrates the near-zone electric field in the vicinity of the periodic slabs. The total electric field is sampled on the $x-z$ plane inside and outside the object. In addition to the complete plot, inner and outer problems are considered separately. This is achieved by allowing the equivalent currents to radiate into two different homogeneous 
media with the inner and outer electrical parameters are assumed everywhere. For the inner problem, fields outside the object should be zero and any nonzero field is due to numerical errors. Similarly, for the outer problem, fields inside the object should be zero. This information can be used to check the accuracy of numerical solutions for arbitrary objects. As depicted in Fig. 3, unwanted radiations are quite low and outside the visible range $(-10-20 \mathrm{~dB})$. The complete plot is obtained by superposing the inner and outer problems, and illustrates how the dipole interacts with the periodic slabs.

\section{CONCLUSION}

Recently developed surface formulations, namely, CTF and JMCFIE, are used to formulate electromagnetics problems involving lossy dielectric objects. Solutions are performed iteratively using MLFMA. We show that lossy dielectric objects with large conductivity values can be analyzed accurately and efficiently with CTF.

\section{ACKNOWLEDGMENT}

This work was supported by the Centre for Numerical Algorithms and Intelligent Software (EPSRC EP/G036136/1), the Scientific and Technical Research Council of Turkey (TUBITAK) under the Research Grant 110E268, by the Turkish Academy of Sciences in the framework of the Young Scientist Award Program (LG/TUBA-GEBIP/2002-1-12), and by contracts from ASELSAN and SSM. Özgür Ergül was also supported by a Research Starter Grant provided by the Faculty of Science at the University of Strathclyde.

\section{REFERENCES}

[1] T. K. Wu and L. L. Tsai, "Scattering from arbitrarily-shaped lossy dielectric bodies of revolution," Radio Sci., vol. 12, pp. 709-718, Sep.Oct. 1977.

[2] S. M. Rao, C. C. Cha, R. L. Cravey, and D. Wilkes, "Electromagnetic scattering from arbitrary shaped conducting bodies coated with lossy materials of arbitrary thickness," IEEE Trans. Antennas Propag., vol. 39, pp. 627-631, no. 5, May 1991.

[3] X.-Q. Sheng, J.-M. Jin, J. Song, W. C. Chew, and C.-C. Lu, "Solution of combined-field integral equation using multilevel fast multipole algorithm for scattering by homogeneous bodies," IEEE Trans. Antennas Propag., vol. 46, no. 11, pp. 1718-1726, Nov. 1998.

[4] K. C. Donepudi, J.-M. Jin, and W. C. Chew, "A higher order multilevel fast multipole algorithm for scattering from mixed conducting/dielectric bodies," IEEE Trans. Antennas Propag., vol. 51, no. 10, pp. 2814-2821, Oct. 2003.

[5] P. Ylä-Oijala and M. Taskinen, "Application of combined field integral equation for electromagnetic scattering by dielectric and composite objects," IEEE Trans. Antennas Propagat., vol. 53, no. 3, pp. 11681173, Mar. 2005.

[6] Y.-H. Chu and W. C. Chew, "A robust surface-integral-equation formulation for conductive media," Microw. Opt. Technol. Lett., vol. 46, no. 2, pp. 109-114, Jul. 2005.

[7] P. Ylä-Oijala, M. Taskinen, and S. Järvenpää, "Surface integral equation formulations for solving electromagnetic scattering problems with iterative methods," Radio Science, vol. 40, RS6002, doi:10.1029/2004RS003169, Nov. 2005.

[8] Ö. Ergül and L. Gürel, "Comparison of integral-equation formulations for the fast and accurate solution of scattering problems involving dielectric objects with the multilevel fast multipole algorithm," IEEE Trans. Antennas Propag., vol. 57, no. 1, pp. 176-187, Jan. 2009.

[9] Ö. Ergül and L. Gürel, "Efficient solution of the electric and magnetic current combined-field integral equation with the multilevel fast multipole algorithm and block-diagonal preconditioning," Radio Sci., vol. 44, RS6001, doi:10.1029/2009RS004143, Nov. 2009.
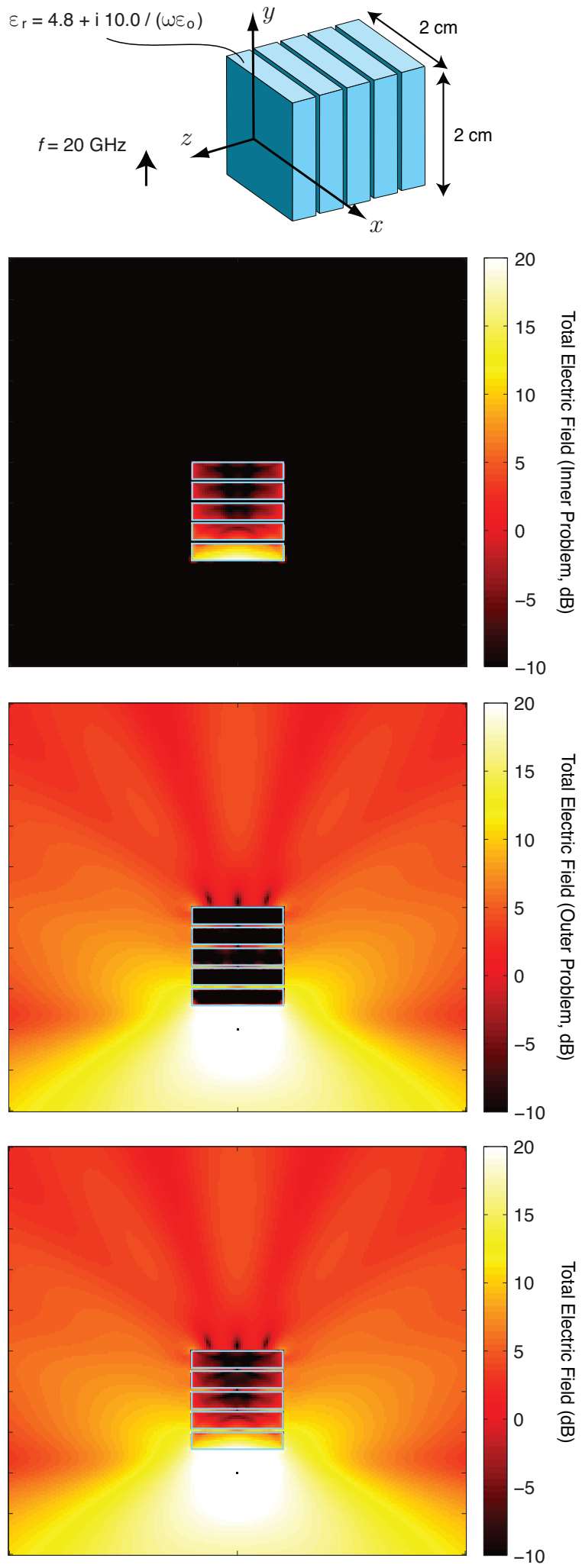

Fig. 3. The total electric field on the $x-z$ plane in the vicinity of a structure involving periodic slabs formulated with CTF. The relative permittivity and the conductivity of the object is 4.8 and $10.0 \mathrm{~S} / \mathrm{m}$, respectively. In addition to the complete plot, near-zone fields are depicted for the inner and outer problems. 